\title{
X-ray Spectra Calculations for Inspection of Semiconductors with Different Target/Filter Combinations Using MCNPX
}

Jeong-Ho Kim ${ }^{1}$, Hye-Min Park ${ }^{1}$, Ki-Hyun Park ${ }^{1}$, Chan-Jong Park ${ }^{1}$, Seung-Ho Lee ${ }^{2}$, Dong-Sung Kim² ${ }^{2}$ Joong-Suk Youn $^{2}$, Seing-Won Jeon ${ }^{2}$ and Koan-Sik Joo ${ }^{1}$

1. Department of Physics, University of Myongji, Yongin KS009, Korea

2. SEC111, Saneop-ro, 155 beon-gil, Gwonseon-gu, Suwon KS002, Korea

\begin{abstract}
X-rays are commonly used for inspecting semiconductors. However, excessive radiation dose could damage semiconductors. Therefore, unnecessary exposure needs to be reduced. The ray quality, which is influenced by the tube voltage and filter, determines the exposure. We designed an X-ray tube for inspecting semiconductors with different target/filter combinations and calculated X-ray spectra using the MCNPX (Monte Carlo n-particle extended) code. The target material was W, and the filters were made of Mo, W, and Zr. The W/W combination showed the lowest flux. The MCNPX code can reduce the development time and cost of the target/filter combination for inspecting semiconductors.
\end{abstract}

Key words: Inspection, semiconductor, damage, filter, MCNPX.

\section{Introduction}

Semiconductor manufacturing technologies are continually being researched to improve the performance and degree of integration and to reduce the size of semiconductors. Semiconductors are often very small, and therefore, inspection cannot be performed using the naked eye below a certain size. To overcome this problem, $\mathrm{X}$-rays are used as a nondestructive defect inspection method. For example, X-ray laminography, a technique that captures tomographic photos of a sample multilayer by using the data obtained from X-rays and a detector, is used for nondestructive inspection. However, recent studies on the damage caused to semiconductors by the TID (total ionizing dose) effect of BJTs (bipolar junction transistors) raised the issue of low-energy $\mathrm{X}$-rays causing damage to semiconductors [1]. TID is mostly caused by charge trapping when electron holes form between silicon and silicon dioxide in semiconductors.

Corresponding author: Koan-Sik Joo, Ph.D., professor, research field: physics.
Threshold voltage changes and leakage current increases when TID occurs and MOSFET is used [2]. Therefore, methods to reduce damage need to be studied. Generally, the ray quality in radiation imaging is an important factor that determines the amount of radiation exposure; it is affected by the tube voltage and added filter [3]. The inverse proportionality of the photoelectric effect to the cube of the energy of incident photons means that if a photon's energy is increased slightly, the photoelectric effect will decrease sharply. Therefore, to minimize the dose, a high $\mathrm{kVp}$ technique that increases the $\mathrm{X}$-ray energy is recommended. However, this technique leads to another problem: the Compton scattering interaction increases when photoelectric absorption decreases, and the scattered photons deteriorate the image quality. This is because when a large photoelectric effect occurs, attenuation is severe, and when a large Compton reaction occurs, attenuation decreases [4]. In general, various types of added filters that can effectively reduce the 
unnecessary energy region must be developed. However, fabricating an added filter and evaluating its properties incurs considerable time and cost. The MCNPX (Monte Carlo n-particle extended) code is a powerful tool for studying and optimizing ionizing radiation systems. In this study, the X-ray spectra for different target-filter combinations were calculated by the Monte Carlo method using the MCNPX code.

\section{Experimental Setup}

Fig. 1 shows the simplified geometry configuration of the X-ray tube used for inspection. The X-ray tube used lead as a shielding material, and its length and diameter were 60 and $15 \mathrm{~cm}$, respectively. The outside of the X-ray tube was set to be air and the inside, vacuum. The target material used was $W$. The filter was made from Mo, W, and $\mathrm{Zr}$. The position at which the target and electrons collide was set to be the origin point $(0,0,0)$. The distance between the target and the filter was $76 \mathrm{~cm}$. The target had a diameter and thickness of $8 \mathrm{~cm}$ and $25 \mu \mathrm{m}$, respectively. Table 1 lists the characteristics of the target and filter materials.

Mo has a similar K-absorption edge as $\mathrm{Zr}$. W has a higher-energy K-absorption edge compared to the

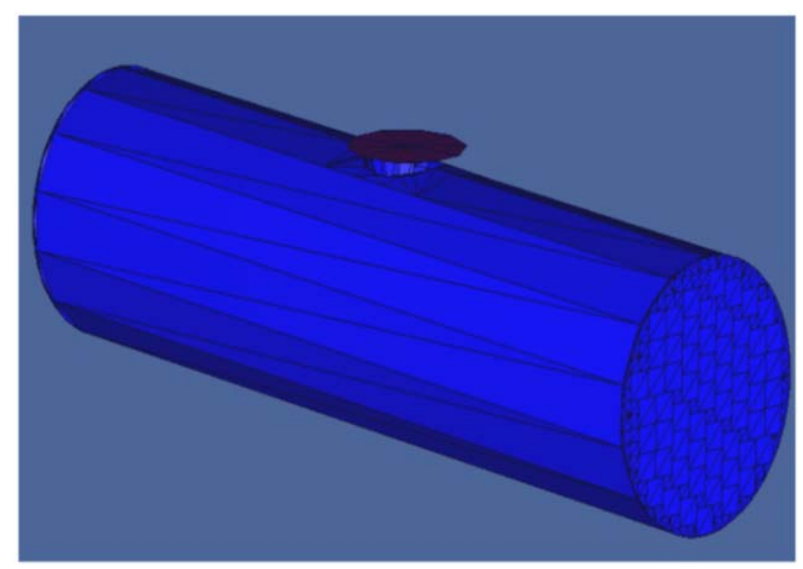

Fig. 1 Geometry configuration of X-ray tube. other materials. The maximum tube voltage was set as $50,80,10$, and $120 \mathrm{keV}$ (erg $=0.028$ ), and the tube current was $100 \mathrm{~mA}$. Because MCNPX outputs a value corresponding to one electron, the tube current was coded using Eq. (1) (fm 2.250e21). The mode was e and $\mathrm{p}$ (mode e $\mathrm{p}$ ). The particle number was 3 (electron, par $=3$ ). The distance between the target and the electron was $5 \mathrm{~cm}($ pos $=500)$. Tally cards were used to specify the type of information that the user wanted to obtain from the Monte Carlo calculation. The information included the current across a surface, flux at a point, and heating in a region [5]. Table 2 shows a description of the tally card. To find the value of the flux averaged over a cell, we used *F4 tally (*F4:p 6). Further, to calculate the X-ray spectra, we used the e code (e4 1e-10 999i 0.2); in this case, the energy of the desired region can be divided into the desired number. NPS denotes the number of runs. To reduce the error $(<3 \%)$, NPS was set to $1 \mathrm{e} 8$.

fm code at $100 \mathrm{~mA}=$ Tube current $\times$ Number of electrons per $1 \mathrm{~A} \times 3600=100 \mathrm{~mA} \times 6.250 \times 1018 / \mathrm{A}$ $\times 3600=2.250 \times 1021$

Fig. 2 shows the Cd-Te X-ray spectrometer used for $\mathrm{X}$-ray spectrum measurement in this study. Because

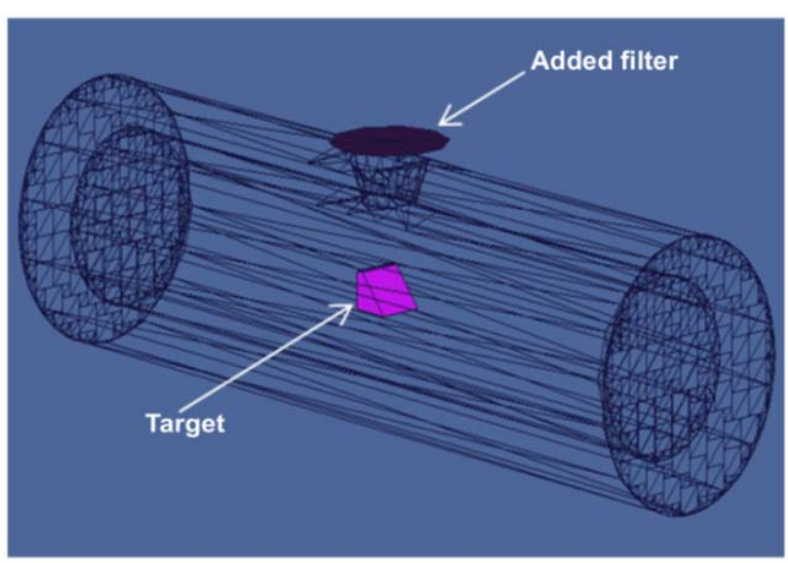

Table 1 Characteristics of the target and filter materials.

\begin{tabular}{llll}
\hline & Atomic number & Density $\left(\mathrm{g} / \mathrm{cm}^{3}\right)$ & K-edge $(\mathrm{keV})$ \\
\hline Tungsten & 74 & 19.25 & 69.51 \\
Molybdenum & 42 & 10.28 & 20.00 \\
Zirconium & 40 & 6.52 & 17.99 \\
\hline
\end{tabular}


Table 2 Description of tally card.

\begin{tabular}{|l|l|l|l|}
\hline Tally type & Tally description & Fn units & *Fn units \\
\hline F1 $(\mathrm{n}, \mathrm{p}, \mathrm{e})$ & Current integrated over a surface & particles & $\mathrm{MeV}$ \\
\hline F2 $(\mathrm{n}, \mathrm{p}, \mathrm{e})$ & Flux averaged over a surface & particles $/ \mathrm{cm}^{2}$ & $\mathrm{MeV} / \mathrm{cm}^{2}$ \\
\hline F4 $(\mathrm{n}, \mathrm{p}, \mathrm{e})$ & Flux averaged over a cell & particles $/ \mathrm{cm}^{2}$ & $\mathrm{MeV} / \mathrm{cm}^{2}$ \\
\hline F5 $(\mathrm{n}, \mathrm{p})$ & Flux at a point or ring detector & particles $/ \mathrm{cm}^{2}$ & $\mathrm{MeV} / \mathrm{cm}^{2}$ \\
\hline F6 $(\mathrm{n}, \mathrm{p})$ & Energy deposition averaged over a cell & $\mathrm{MeV} / \mathrm{g}$ & $\mathrm{Jerks} / \mathrm{g}$ \\
\hline F7 $(\mathrm{n})$ & Fission energy deposition averaged over a cell & $\mathrm{MeV} / \mathrm{g}$ & Jerks $/ \mathrm{g}$ \\
\hline F8 $(\mathrm{p})$ & Energy distribution of pulses created in a detector by radiation & Pulse & $\mathrm{MeV}$ \\
\hline
\end{tabular}

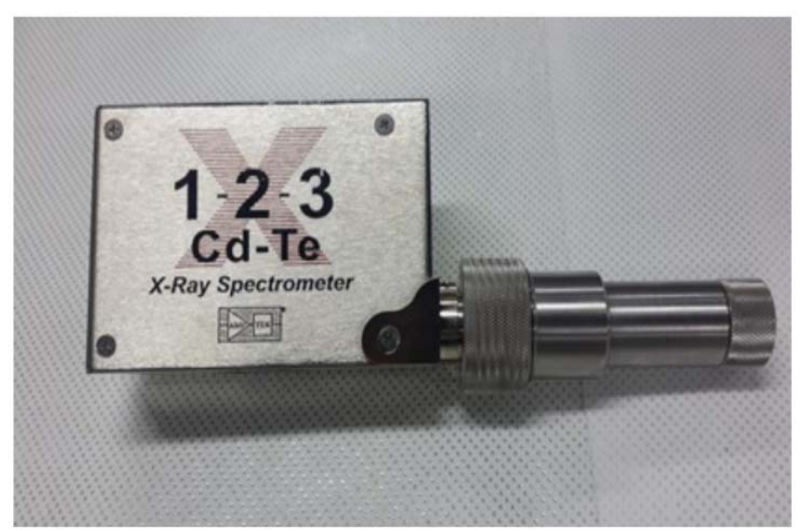

Fig. 2 Cd-Te X-ray spectrometer.

this spectrometer works at room temperature and has high mass absorption coefficient when exposed to the $\mathrm{X}$-rays of a sample, as a semiconductor detector, it affords greater advantages than other $\mathrm{Si}$ and $\mathrm{Ge}$ semiconductor detectors, which have different photoelectric absorption probabilities.

\section{Experimental Results}

Fig. 3 shows the X-ray spectra without the filter for energies of $50,80,100$, and $120 \mathrm{keV}$. The characteristic X-ray peaks of $\mathrm{W}$ are very clear in these spectra. The maximum energy of the $\mathrm{X}$-ray spectrum was confirmed to be $50,80,100$, and $120 \mathrm{keV}$.

Fig. 4 shows the X-ray spectrum of the inspected equipment for tube voltage of $120 \mathrm{keV}$ and another $\mathrm{X}$-ray spectrum with a filter to reduce the dose. The characteristic peaks of $\mathrm{W}$ and of each filter were observed in all spectra. Compared to the case without a filter, the absorbed dose in air decreased by $\sim 25.9 \%$, $24.9 \%$, and $26.9 \%$ when $\mathrm{Mo}, \mathrm{Zr}$, and $\mathrm{W}$ filters were used. Furthermore, compared to the case without a filter in the energy range below $40 \mathrm{keV}$, the absorbed dose in air decreased by $\sim 35.4 \%, 33.2 \%$, and $38.0 \%$ when Mo, Zr, and W filters were used. Fig. 5 shows the MCNPX value at $50 \mathrm{keV}$ as well as the values measured using the real inspection equipment. The computer simulation results show that the absorbed dose in air decreased by $\sim 47.3 \%$ with the Mo filter

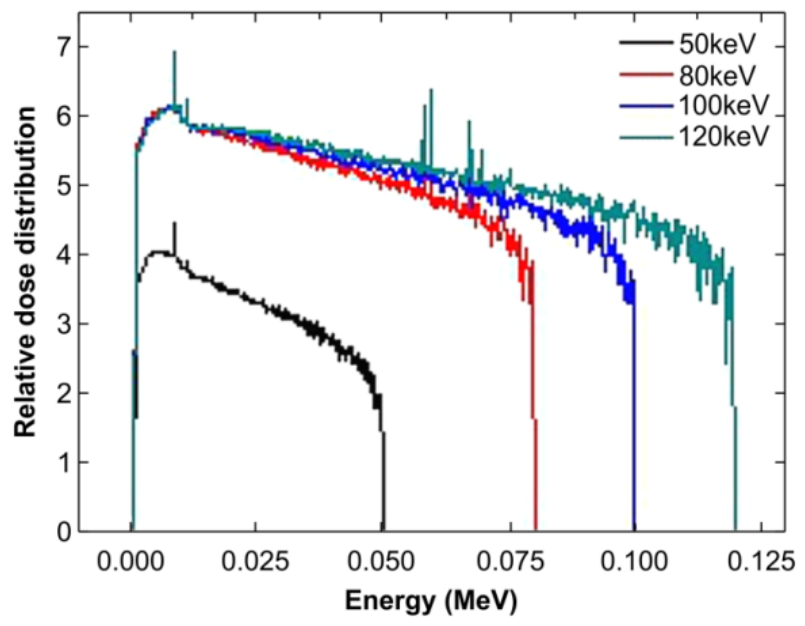

Fig. 3 X-ray spectra without filter for 50, 80, 100, 120 keV.

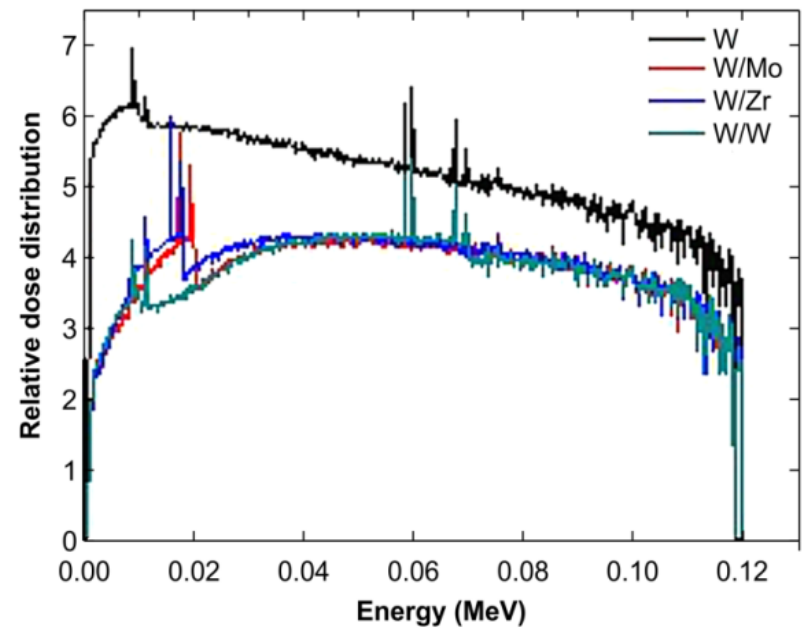

Fig. 4 X-ray spectrum $(120 \mathrm{keV})$ of the inspection equipment designed using MCNPX. 

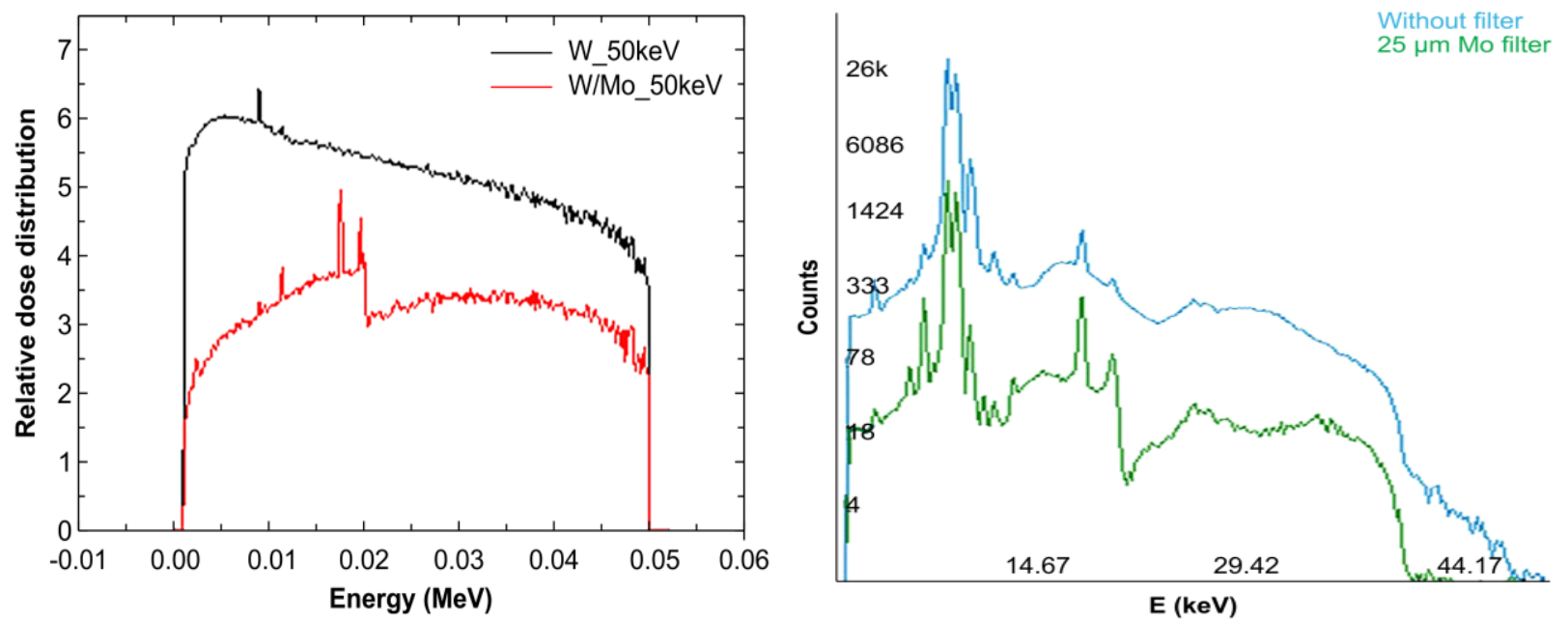

Fig. 5 X-ray spectrum (50 keV) of the inspection equipment designed with MCNPX and that of the actual inspection equipment.

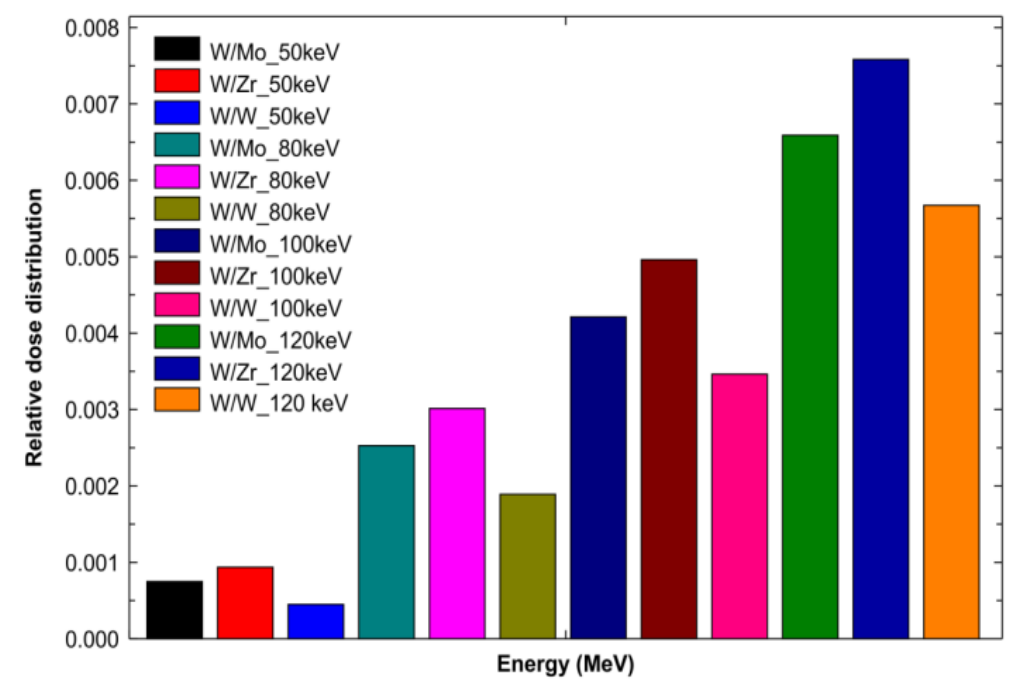

Fig. 6 Relative dose distribution for each filter.

compared to the case without the Mo filter. The values measured with $\mathrm{Cd}$-Te show that the absorbed dose in air decreased by $\sim 40.1 \%$ with the Mo filter compared to the case without the Mo filter.

Fig. 6 shows the absorbed dose when Mo, Zr, and W filters were used at energies of 50, 80, 100, and 120 $\mathrm{keV}$. The results confirmed that the absorbed dose was the lowest at every tube voltage when the $\mathrm{W}$ filter was used.

\section{Conclusions}

This research used MCNPX to design various filters. The MCNPX values were compared with the measured values with the aim of reducing the damage caused to semiconductors during inspection. The results showed that the inspection equipment using a $\mathrm{W}$ filter absorbed the most dose at tube voltages of 50, 80, 100, and $120 \mathrm{keV}$. Furthermore, the MCNPX values showed $47.3 \%$ decay rate at $50 \mathrm{keV}$; in comparison, the measured values which showed $40.1 \%$ decay rate, indicating an error of $7.2 \%$. Based on this study, equipment that reduces damage caused to semiconductors while enabling better imaging can be developed if more various filters are designed and manufactured to evaluate the resulting doses and images. 


\section{Acknowledgments}

This study was supported by Korea Evaluation Institute of Industrial Technology (No. 10045844).

\section{References}

[1] Kim, D. S. 2015. "Radiation Damage of Semiconductor Device by X-ray." Journal of Radiation Protection and Research 40: 110-7.

[2] Daniel, M. F. 2013. "Total Ionizing Effects in MOS and Low-Dose-Rate-Sensitive Linear-Bipolar
Devices." IEEE Transactions on Nuclear Science 60: 1706-30.

[3] Cho, W. I. 2013. "Change of Dose Exposure and Improvement of Image Quality by Additional Filtration in Mammography." Journal of Radiological Protection 33: 78-90.

[4] Nishino, T. K. 2005. "Thickness of Molybdenum Filter and Squared Contrast-to-Noise' Ratio per Dose for Digital Mammography." American Journal of Roentgenology 185: 960-3.

[5] Pelowitz, D. B., et al. "MCNPX User's Manual." version 2.5.0. 2005 LA-CP-05-0369. 